Open Access

\title{
A proliferation saturation index to predict radiation response and personalize radiotherapy fractionation
}

Sotiris Prokopiou ${ }^{1}$, Eduardo G. Moros ${ }^{2,3}$, Jan Poleszczuk ${ }^{1}$, Jimmy Caudell ${ }^{2}$, Javier F. Torres-Roca ${ }^{2}$, Kujtim Latifi ${ }^{2}$, Jae K. Lee ${ }^{4}$, Robert Myerson ${ }^{5}$, Louis B. Harrison ${ }^{2}$ and Heiko Enderling ${ }^{1 *}$

\begin{abstract}
Background: Although altered protocols that challenge conventional radiation fractionation have been tested in prospective clinical trials, we still have limited understanding of how to select the most appropriate fractionation schedule for individual patients. Currently, the prescription of definitive radiotherapy is based on the primary site and stage, without regard to patient-specific tumor or host factors that may influence outcome. We hypothesize that the proportion of radiosensitive proliferating cells is dependent on the saturation of the tumor carrying capacity. This may serve as a prognostic factor for personalized radiotherapy (RT) fractionation.
\end{abstract}

Methods: We introduce a proliferation saturation index (PSI), which is defined as the ratio of tumor volume to the host-influenced tumor carrying capacity. Carrying capacity is as a conceptual measure of the maximum volume that can be supported by the current tumor environment including oxygen and nutrient availability, immune surveillance and acidity. PSI is estimated from two temporally separated routine pre-radiotherapy computed tomography scans and a deterministic logistic tumor growth model. We introduce the patient-specific pre-treatment PSI into a model of tumor growth and radiotherapy response, and fit the model to retrospective data of four non-small cell lung cancer patients treated exclusively with standard fractionation. We then simulate both a clinical trial hyperfractionation protocol and daily fractionations, with equal biologically effective dose, to compare tumor volume reduction as a function of pretreatment PSI.

Results: With tumor doubling time and radiosensitivity assumed constant across patients, a patient-specific pretreatment PSI is sufficient to fit individual patient response data $\left(R^{2}=0.98\right)$. PSI varies greatly between patients (coefficient of variation $>128 \%$ ) and correlates inversely with radiotherapy response. For this study, our simulations suggest that only patients with intermediate PSI (0.45-0.9) are likely to truly benefit from hyperfractionation. For up to $20 \%$ uncertainties in tumor growth rate, radiosensitivity, and noise in radiological data, the absolute estimation error of pretreatment PSI is $<10 \%$ for more than $75 \%$ of patients.

Conclusions: Routine radiological images can be used to calculate individual PSI, which may serve as a prognostic factor for radiation response. This provides a new paradigm and rationale to select personalized RT dose-fractionation.

Keywords: Proliferation saturation index, Personalized radiotherapy, Logistic tumor growth, Mathematical model, Hyperfractionation

\footnotetext{
* Correspondence: Heiko.Enderling@Moffitt.org

${ }^{1}$ Departments of Integrated Mathematical Oncology, H. Lee Moffitt Cancer

Center \& Research Institute, 12902 Magnolia Drive, Tampa, FL 33612, USA

Full list of author information is available at the end of the article
} 


\section{Background}

Recent advances in radiation oncology have largely focused on the physical characteristics of radiation including beam quality and delivery. Typically, tumor and normal tissue anatomy/geometry are only used as the main parameters with which to enhance therapeutic ratios [1-5]. Adaptive radiotherapy and image-guided radiotherapy have been suggested to primarily re-shape the target volume based on changes in tumor volume or position [1-4], rather than as a methodology to adapt to changes in the intrinsic tumor-host biology. Inroads have been made in combining intensity-modulated radiation therapy with functional imaging, such as by $\left[{ }^{18} \mathrm{~F}\right]$-fluoromisonidazole positron emission tomography (FMISO-PET) or dynamic contrast-enhanced magnetic resonance imaging, for radiation planning and response assessment to direct higher doses of radiation to areas with increased radioresistance [5-8]. In conventional clinical practice, however, most patients treated with definitive radiotherapy receive a similar dose and fractionation scheme based upon primary site and American Joint Committee on Cancer (AJCC) TNM stage (Tumor size, lymph Node involvement, Metastasis presence).

Different fractionation protocols have been tested in prospective clinical trials $[9,10]$. Alternative radiation fractionation protocols may improve outcome for some patients but worsen outcome for others. It is important that we begin to understand which tumors respond better to altered fractionation, and how to select the most appropriate fractionation schedule for an individual patient. Innovative models that are based upon cell biology and interactions of the tumor with its unique environment could forecast individual radiation response and justify recommendation of either standard of care or alternative radiation fractionation on a per patient basis.

Tumors grow within a host tissue that both facilitates progression by supplying nutrients and growth factors $[11,12]$, and inhibits it through physical constraints [13] and immune surveillance $[14,15]$. Since many of these factors vary widely across patients, we introduce the concept of tumor carrying capacity as the maximum tumor volume that is achievable in the patient-specific tumor environment, and saturation of tumor proliferation as the tumor approaches its carrying capacity. We propose a non-invasive radiomics measurement of patient-specific carrying capacity and proliferation saturation, which may ultimately help designing more personalized approaches to radiotherapy.

Tumors are composites of proliferating and growth arrested cells. Their respective proportions at individual times during radiation contribute to the populationlevel response, in addition to radiation beam and protocol parameters and host tissue properties. In multi-compartment mathematical models that distinguish between cycling and growth-arrested cells, proliferation and oxygenation status-dependent radiation response could be simulated on the cellular level $[16,17]$. Tumor growth in vivo can be approximated by logistic dynamics [18]. Initial exponential growth at low cell densities when most cells have access to ample resources decelerates when cells at the core of the tumor become growtharrested, mainly due to limited space and exhausted intratumoral nutrient supply as resources are consumed by cells closer to the tumor surface $[19,20]$. This established the notion of a tumor carrying capacity $(\mathrm{K})$ as the maximum tumor volume $(\mathrm{V})$ that can be supported by a given environment. A tumor carrying capacity may change depending on the oxygen and nutrient supply through tissue vascularization [21], removal of metabolic waste products [22], and evasion of immune surveillance [14]. Greater oxygen supply and removal of metabolic waste increases tumor carrying capacity; in contrast, infiltration of tumor specific cytotoxic $\mathrm{T}$ lymphocytes exemplifies a reduction of carrying capacity. Hence, the tumor volume-to-carrying capacity ratio $(\mathrm{V} / \mathrm{K})$ describes the saturation of tumor cell proliferation at the population level as the tumor approaches its carrying capacity, and as such is deemed the Proliferation Saturation Index (PSI). The PSI at any time reflects the history of the reciprocal changes of a tumor and its environment - and thus can be expected to be patient-specific. Tumor volumes close to their carrying capacity, i.e., with a high PSI, are here assumed to have only a small proportion of proliferating cells that are most sensitive to radiation-induced damage. We therefore hypothesize that a patient-specific PSI may serve as a novel prognostic factor for radiotherapy response.

\section{Methods}

\section{Tumor radiation response model and Proliferation} Saturation Index (PSI)

Logistic tumor growth is modeled as a deterministic ordinary differential equation between radiation doses

$$
\frac{\mathrm{dV}}{\mathrm{dt}}=\lambda \mathrm{V}(1-\mathrm{PSI})
$$

where PSI is the tumor volume-to-carrying capacity ratio $(\mathrm{V} / \mathrm{K})$, and $\lambda=\frac{\ln 2}{T_{\text {eff }}}$ is the intrinsic tumor growth rate, with the effective tumor doubling time $\mathrm{T}_{\text {eff }}$ being a composite of the potential doubling time, $\mathrm{T}_{\mathrm{POT}}$, reduced by the cell loss fraction, $\phi$ [23]. $\mathrm{T}_{\text {eff }}$ is assumed to be intrinsic and time independent. Radiation response after each application of a single dose $d$ is modeled as an instantaneous volume change $\mathrm{V}_{\text {postiR }}=\mathrm{V}-\gamma_{d} \mathrm{~V}\left(1-\frac{\mathrm{V}}{\mathrm{K}}\right)$ at discrete times during irradiation in the considered treatment protocols (standard of care: once a day [q.d.] at 9 am, no treatment on the weekend; hyperfractionation twice a day [b.i.d] at 9 am and $3 \mathrm{pm}$; no weekend), where $\gamma_{d}=1-e^{-\left(\alpha d^{\circ}+{ }^{\circ} \beta d^{2}\right)}$ 
represents radiation-induced death following the linearquadratic model [24, 25]. In this form, radiation-induced cell death is only considered for proliferating cells, while quiescent and hypoxic cells are assumed radioresistant. Tumor growth is modeled following (Eqn. 1) between radiation fractions. While other biological effects are without doubt at play before, during and after RT, longitudinal measurements of these effects are currently impossible and thus intentionally not considered explicitly. However, changes in tumor growth rate such as accelerated repopulation as well as other proliferation stimulating radiation effects (re-oxygenation, re-distribution in the cell cycle) are inherent to the logistic growth and RT model: as the tumor volume shrinks, V/K and thus PSI decreases, which in turn reduces the extrinsically enforced pre-treatment reduction in proliferation. It follows from (Eqn. 1) that larger PSI implies a low proliferating cell fraction and thus treatment refractory tumors, whereas tumors with lower PSI are more proliferative and thus more radiosensitive (Fig. 1a), which is in line with the established positive correlation between proliferation rate and radiosensitivity [18]. Therefore, two patients that present with similar tumor volume could have a different tumor environmental conditions and thus different PSI, which results in different responses to the same RT protocol (Fig. 1b).

Here we assume that the tumor carrying capacity is constant during the 5-7 week course of treatment. This is, of course, a gross oversimplification of the underlying biology, but without longitudinal measurements of carrying capacity biomarkers, calibration of dynamic carrying capacity changes during RT is impossible and would introduce additional uncertainty. The model was simulated in MATLAB using the analytical expression for the solution of (Eqn. 1) (MATLAB R2013b, The MathWorks Inc., Natick, MA).

\section{Prospective estimation of patient-specific pretreatment PSI}

The analytical solution of pretreatment tumor growth (Eqn. 1) is given by $\mathrm{V}=\frac{\mathrm{K} \times \mathrm{V}(0) \times \mathrm{e}^{\lambda t}}{\mathrm{~K}+\mathrm{V}(0) \times\left(\mathrm{e}^{\lambda t}-1\right)}$, with $\mathrm{V}(0)$ being the initial tumor volume at time $t=0$. From two different radiological scans, routinely taken at diagnosis and at radiotherapy treatment planning simulation $(\Delta t=45$ days in private hospitals [26]), we obtain two distinct tumor volumes $\left(\mathrm{V}(0)=\mathrm{V}_{\text {diagnosis }}\right.$ and $\mathrm{V}=\mathrm{V}_{\text {simula- }}$ tion) along the logistic growth trajectory. Then, the analytical solution of the logistic model can be solved explicitly for $\mathrm{K}$, giving the following analytic expression for pretreatment PSI value.

$$
\text { PSI }=\frac{\mathrm{V}_{\text {diagnosis }} \times \mathrm{e}^{\lambda \Delta \mathrm{t}}-\mathrm{Vsimulation}}{\mathrm{V}_{\text {diagnosis }} \times\left(\mathrm{e}^{\lambda \Delta \mathrm{t}}-1\right)}
$$

\section{Data fitting}

Thirteen longitudinal tumor volume measurements across four NSCLC patients treated with fractionated RT (2 Gy x 30 fractions) were taken from the literature [27]. Tumor volumes are available at the beginning of treatment $\left(\mathrm{V}_{\text {simulation }}=\{7.6,27.4,97.7,189.3\} \mathrm{cm}^{3}\right)$ and at least two subsequent RT fractionations for each patient. We assume that the variations in intrinsic effective tumor growth rate $\lambda$ and radiosensitivity $\gamma_{2 \mathrm{~Gy}}$ are negligible between patients compared to the variation in individual carrying capacity $\mathrm{K}$, given the spread of tumor volumes
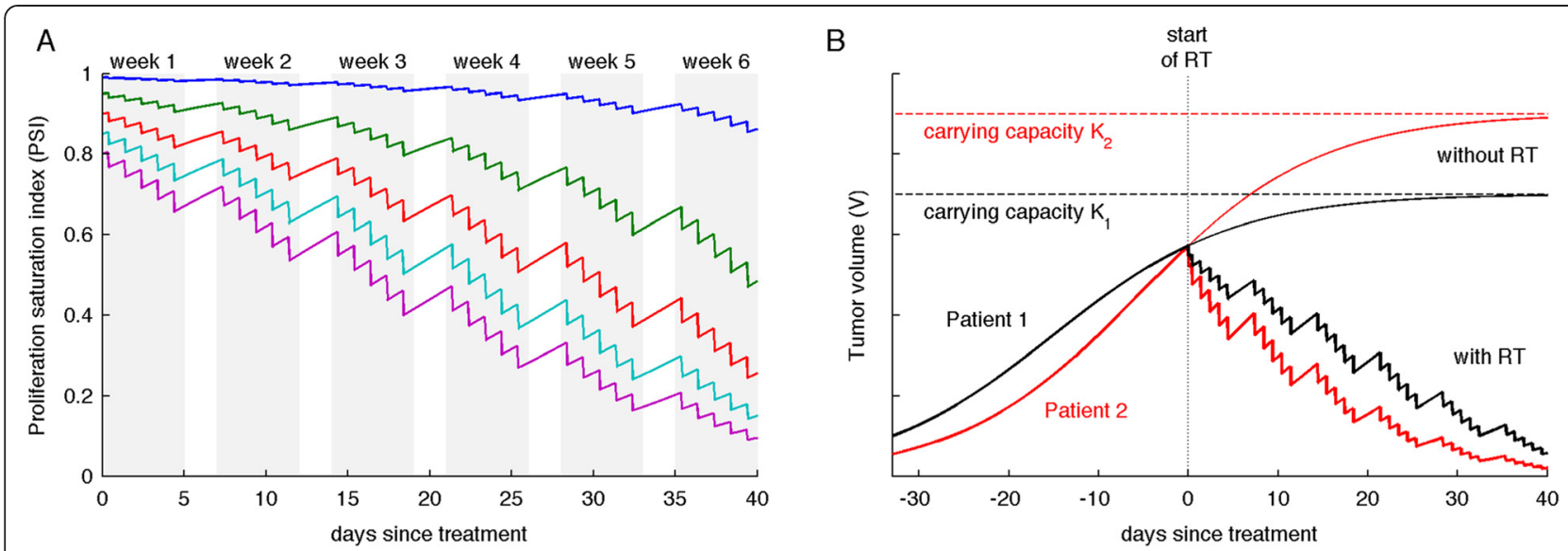

Fig. 1 Pretreatment proliferation saturation index (PSI) determines RT response. a Response for tumors with different initial PSIs to standard of care RT ( $2 \mathrm{~Gy} \times 30$; q.d. 9 am, no weekend) calculated with Eqn. $1 ; \lambda=0.1, \gamma_{2 \mathrm{G}}=0.25$. Each curve shows the dynamics response during six weeks of therapy with different initial PSI. b Two patients with identical tumor volume but different PSI at treatment beginning (day 0) exhibit different reduction in tumor volume after standard of care RT ( $2 \mathrm{~Gy} \times 30$; q.d. 9 am, no weekend). Calculated with Eqn. $1 ; \lambda=0.1, \gamma_{2 G y}=0.25$ 
across two or three orders of magnitude. We therefore estimate constant $\lambda$ and $\gamma_{2 \text { Gy }}$ values for all patients, and individual pretreatment proliferation saturation indices $\left(\mathrm{PSI}_{\mathrm{s}}\right.$ ).

We utilize a genetic algorithm that mimics the processes of evolution and natural selection [28] to derive a combination of parameters that best fits patient data. We generate an initial 'population' ( $N=500$ 'individuals') of parameter sets $\left\{\lambda, \gamma_{2 \mathrm{~Gy}}, \mathrm{PSI}_{1}, \mathrm{PSI}_{2}, \mathrm{PSI}_{3}, \mathrm{PSI}_{4}\right\}$, with each element of each individual parameter set drawn at random from a uniform distribution [0,1]. In each algorithm iteration ('generation') we first evaluate the fitness of each individual in the population by calculating the sum of residuals between data and corresponding simulation results ('cost' C)

$$
\begin{aligned}
& C\left(\left\{\lambda, \gamma_{2 \mathrm{~Gy}}, \mathrm{PSI}_{1}, \mathrm{PSI}_{2}, \mathrm{PSI}_{3}, \mathrm{PSI}_{4}\right\}\right) \\
& =\sum_{k=1}^{4} \sum_{t_{k, i}} \frac{\left(V_{k}\left(t_{k, i}\right)-M\left(\left\{\lambda, \gamma_{2 G \mathrm{~Gy}}, \mathrm{PSI}_{1}, \mathrm{PSI}_{2}, \mathrm{PSI}_{3}, \mathrm{PSI}_{4}\right\}, t_{k, i}\right)\right)^{2}}{V_{\text {simulation }, \mathrm{k}}^{2}}
\end{aligned}
$$

where $t_{k, i}$ represents the time point when tumor volume $V_{k}$ was measured for $\mathrm{kth}$ patient, and $M$ is the simulated tumor volume at that time point. We then select the $50 \%$ fittest individuals (i.e., parameter combinations) that have the smallest calculated cost (Eqn. 3), and discard unfit individuals. To maintain a constant population size $\mathrm{N}$ in subsequent generations, additional individuals are generated using crossover ( $25 \%)$ and mutation (25\%) of survived selected individuals. For crossover, two survived selected individuals are chosen at random to generate a new individual 'offspring', by randomly selecting 'parental' parameter combinations (pairwise mixing). For mutation, a new individual is generated from a survived individual with either $\lambda, \gamma_{2 G y}$ or $\operatorname{PSI}_{i}$ being modified randomly by changing its current value randomly up to $\pm 10 \%$.

The 500 individuals after 500 iterations of the selection, crossover, and mutation procedure of the genetic algorithm (i.e., best sets of parameter combinations with the smallest sum of residuals between data and simulation results) is then refined with a trust-region-reflective algorithm (deterministic, gradient based optimization procedure) implemented in the MATLAB lsqnonlin function (MATLAB R2013b with Optimization Toolbox, The MathWorks Inc., Natick, MA), which uses a quadratic approximation for the minimized function in a neighborhood (trust region) around the current point. In order to avoid finding a parameter set yielding only local minimum in the fitness landscape we reiterated the whole fitting procedure 20 times and compared the resulting parameter sets.

\section{Alternative radiotherapy protocols}

From the estimation of radiation-induced cell death, $\gamma_{2 \text { Gy }}$ we can approximate the radiosensitivity parameters $\alpha$ and $\beta$ to derive $\gamma_{\mathrm{d}}$ for any dose $d$ using the linear-quadratic model $\gamma_{d}=1-e^{-\left(\alpha \mathrm{d}+\beta \mathrm{d}^{2}\right)}$.

\section{Virtual patient cohort}

We create a cohort of $n=1,000$ in silico virtual patients $P_{i}$ for which we randomly assign tumor growth rate $\lambda_{i} \in$ [ $\lambda *(1-\mathrm{x} \%), \lambda *(1+\mathrm{x} \%)]$ and radiation-induced cell death $\gamma_{2 \mathrm{~Gy}_{i}} \in\left[\gamma_{2 \mathrm{~Gy}} *(1-\mathrm{x} \%), \gamma_{2 \mathrm{~Gy}} *(1+\mathrm{x} \%)\right]$ from uniform distributions, where $\mathrm{x}$ represents the level of uncertainty (width of the uniform distribution support). For each virtual patient we randomly assign tumor volume and PSI at beginning of treatment, and simulate tumor volume reduction after standard of care 2 Gy x 30 fractionation using Eqn. 1. We calculate the coefficient of determination, $R^{2}$, to investigate how different degrees of uncertainty (value of $\mathrm{x}$ ) impact the predictive power of PSI.

\section{Results}

\section{Logistic tumor growth and radiation response model fits} retrospective data

The logistic tumor growth and radiation response model (Eqn. 1) fits retrospective longitudinal patient-specific data from the literature [27] with highest accuracy $\left(R^{2}=0.98\right)$ for $\lambda=0.045 \mathrm{day}^{-1}$ and $\gamma_{2 \mathrm{~Gy}}=0.084$ (Fig. 2). The estimate for $\lambda$ suggests $T_{\text {eff }}=15.4$ days, which indicates a cell loss factor of $\phi=50 \%$ for $\mathrm{T}_{\mathrm{POT}}=7.7$ days for lung

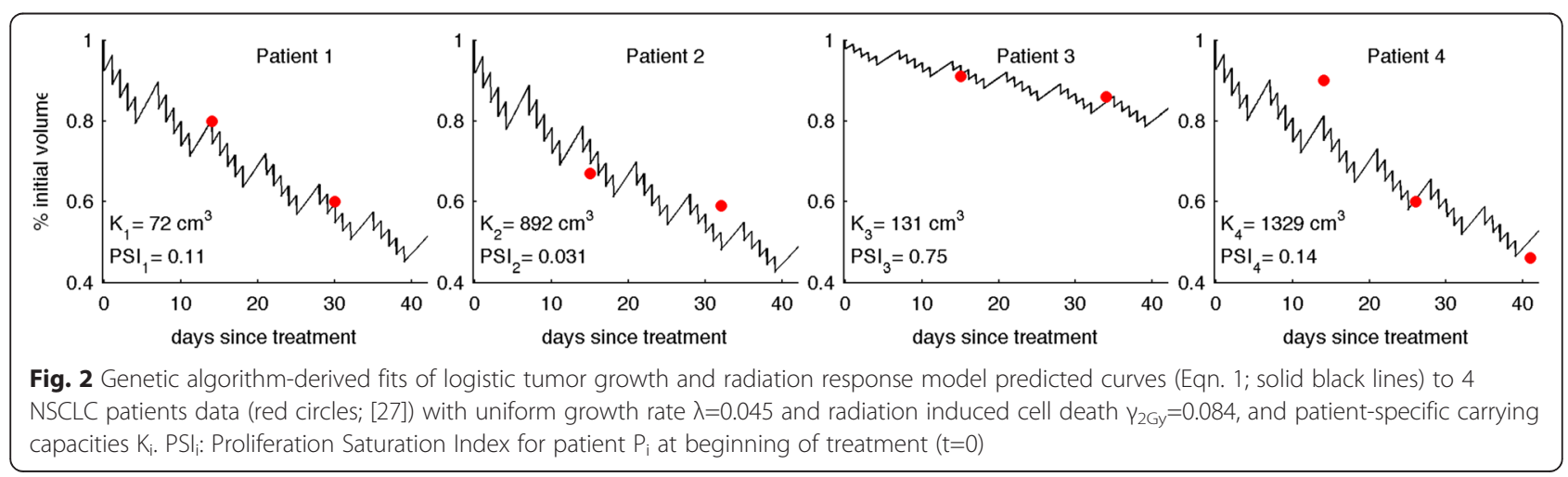


adenocarcinoma [29]. Similar to oropharyngeal cancer cells, fast proliferating NSCLC cells are believed to have a relatively high $\alpha / \beta=20$ [30-33]. With the $\alpha / \beta=20$ approximated from literature, $\gamma_{2 G y}=0.084$ suggests a radiosensitivity parameter $\alpha=0.0487$ and thus $S(2 \mathrm{~Gy})<91.6 \%$, which is similar to that reported for A549 if plated before irradiation (83.3\%; [34]) with additional consideration for senescence and transient cell cycle arrest.

Patient-specific carrying capacities are estimated at $K=\{72,892,131,1329\} \mathrm{cm}^{3}$, with pretreatment PSIs ranging from 0.03 (patient 2) to 0.75 (patient 3) with an average of $0.26 \pm 0.33$ (Fig. 2). Interestingly, although the initial tumor volume of patient 4 is 25 times larger than that of patient 1 , the reduction of tumor volume is comparable in both patients $(53.4 \%$ and $52.1 \%$ ) as their PSIs are similar (0.11 and 0.14). Patient 3 , with an intermediate initial tumor volume but large PSI (0.75), has a significantly smaller reduction of tumor volume (20.4\%). Table 1 summarizes patient-specific tumor volumes $\mathrm{V}_{\mathrm{i}}$, derived carrying capacities $K_{i}$ and proliferation saturation indices $\mathrm{PSI}_{\mathrm{i}}$, and other model parameter values. The minima obtained in each of the 20 independent iterations of the data fitting procedure were indistinguishable ( standard deviation $/$ mean $=6 \times 10^{-6}$ ) with negligible differences between the estimated growth rates and radiosensitivities $\left(\lambda=0.045 \pm 3 \times 10^{-5}\right.$ and $\left.\gamma_{2 \text { Gy }}=0.084 \pm 6 \times 10^{-5}\right)$. The differences in the estimated PSI $_{\mathrm{i}}$ values between independent data fitting iterations did not exceed $10 \%$.

\section{Pretreatment PSI is a prognostic factor for radiation response}

For less than $8.7 \%$ uncertainty in intrinsic tumor parameters, pretreatment PSI serves as a prognostic factor with a high coefficient of determination $\left(R^{2}>0.8\right.$; Fig. $\left.3 a\right)$. $\mathrm{R}^{2}$ falls below 0.6 for $14.1 \%$ uncertainty in $\lambda$ and $\gamma_{2 \mathrm{~Gy}}$, but remains a better prognostic factor for radiation response than tumor growth rate for uncertainty in intrinsic tumor parameters up to around $25 \%$. Pretreatment PSI correlates inversely with tumor volume reduction during RT (Fig. 3b).

Reliable estimation of patient-specific pretreatment PSI must be achievable despite inter-patient variation in tumor growth rate $\lambda$, as well as limitation in radiological image resolution and noise in tumor volume measurements [35].
For each virtual patient $P_{i}$ we reverse calculate exact tumor volume at diagnosis using Eqn. 1 (t-45 days; [26]), and introduce noise of $\pm 5 \%$ in the tumor volume measures at both diagnosis and treatment planning. The absolute error between exact pretreatment PSI and the proposed estimate using Eqn. 2 from noisy input data for different levels of uncertainty in tumor growth rate $\lambda$ is shown in Fig. 3c. For uncertainties less than $20 \%$, the absolute estimation error of pretreatment PSI is $<10 \%$ for more than $75 \%$ of patients.

\section{Pretreatment PSI-dependent response to hyperfractionation}

We consider tumors with fixed estimated radiosensitivity $\alpha=0.045 \mathrm{~Gy}-1$, fixed $\alpha / \beta=20 \mathrm{~Gy}$, and varying pre-treatment PSI. We simulate response to standard of care (2 Gy/fx $\mathrm{x}$ 30; q.d. 9 am; no weekend), and compare final tumor sizes to simulated responses to hyperfractionated treatment with $1.2 \mathrm{~Gy} / \mathrm{fx} \times \mathrm{58}$; b.i.d. (9 am and 3 pm; no weekend), as prescribed in the experimental arm of an RTOG phase III trial in regionally advanced unresectable NSCLC [36]. Model simulations predict an average of $27.6 \%$ improved tumor volume reduction after hyperfractionation for all PSI, due to the larger biologically effective dose (BED) (73.8 Gy $\mathrm{G}_{20}$ vs. $66 \mathrm{~Gy}_{20}$ for standard of care) (Fig. 4a). To demonstrate which patients benefit from hyperfractionation, we compare the clinically applied hyperfractionation protocol to daily fractionation with equal BED (2.21 Gy x 30; q.d. 9 am; no weekend). An improvement in tumor volume reduction of $>5 \%$ is predicted for patients with intermediate PSI (0.45-0.9; Fig. 4b). This demonstrates that a patientspecific PSI may inform which patients are most likely to benefit from alternative radiation fractionation prior to clinical intervention.

\section{Conclusions}

Despite inter-patient variability and the differences in tumor biology across disease sites, radiotherapy is conventionally fractionated at 180-200 cGy daily for 5-7 weeks. Current RT fractionation selection is based on average responses from large historical data sets and clinical trial cohorts without consideration of patientspecific characteristics. The potential uniqueness of each patient at diagnosis due to variation in tumor intrinsic

Table 1 Summary of initial tumor volumes, parameters values and fractionation schemes used for four considered NSCLC patients [27]

\begin{tabular}{|c|c|c|c|c|c|c|}
\hline & $\begin{array}{l}\text { Initial tumor volume } \\
\mathrm{V}(0), \mathrm{cm}^{3}\end{array}$ & $\begin{array}{l}\text { Carrying capacity, } \\
\mathrm{K}, \mathrm{cm}^{3}\end{array}$ & $\begin{array}{l}\text { Proliferation saturation } \\
\text { index, PSI, dimensionless }\end{array}$ & $\begin{array}{l}\text { Growth rate, } \\
\lambda \text {, day }^{-1}\end{array}$ & $\begin{array}{l}\text { Radiosensitivity, } \gamma_{2 G y} \\
\text { dimensionless }\end{array}$ & Fractionation scheme \\
\hline Patient 1 & 7.6 & 72 & 0.11 & \multirow[t]{4}{*}{0.045} & \multirow[t]{4}{*}{0.084} & \multirow{4}{*}{$\begin{array}{l}2 \text { Gy } \times 30 \text {; daily at } 9 \text { am; } \\
\text { weekend break }\end{array}$} \\
\hline Patient 2 & 27.4 & 892 & 0.031 & & & \\
\hline Patient 3 & 97.7 & 131 & 0.75 & & & \\
\hline Patient 4 & 189.3 & 1329 & 0.14 & & & \\
\hline
\end{tabular}



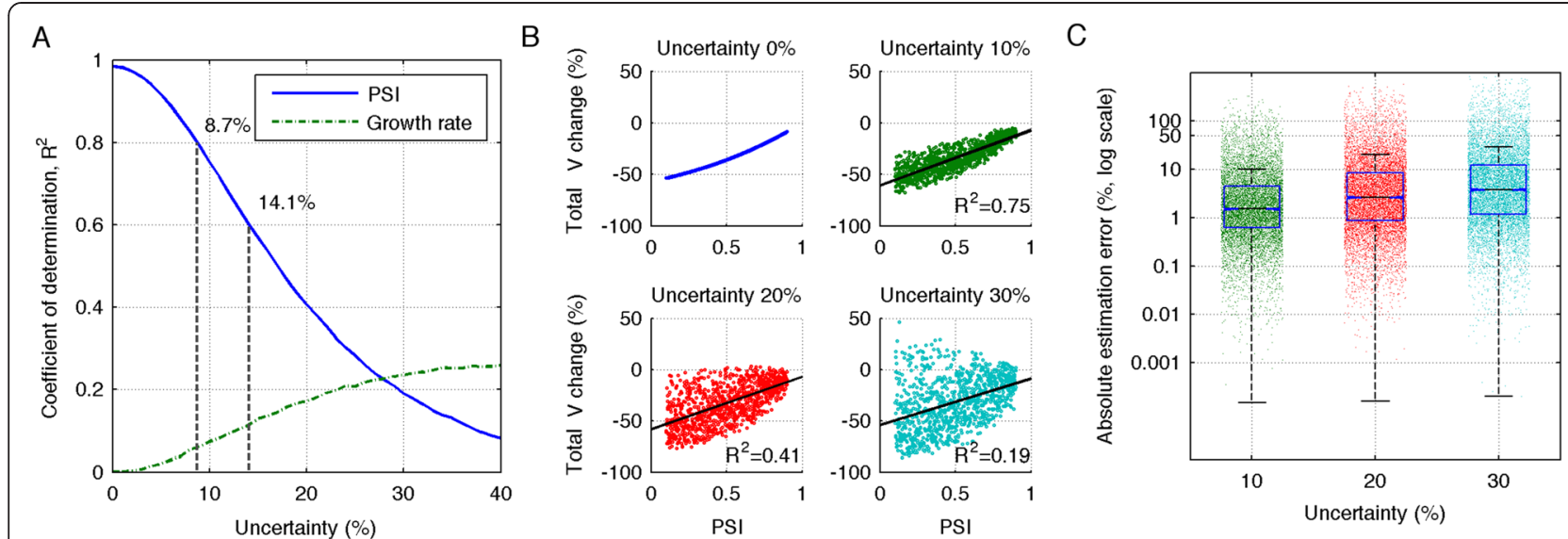

Fig. 3 Pretreatment PSI as a prognostic factor. a Coefficient of determination, $\mathrm{R}^{2}$, for pretreatment PSI and growth rate $\lambda$ as prognostic factors for tumor volume change after RT (2Gy x 30; q.d. 9 am) dependent on percentage of uncertainty in growth rate $\lambda$. $\mathbf{b}$ Predicted tumor volume change as a function of pretreatment PSI, dependent on uncertainty in growth rate $\lambda$. c Error in estimated patient-specific pretreatment PSI using Eq. 2 due to $5 \%$ noise in measured tumor volumes and uncertainty in growth rate $\lambda$ for $N=10,000$ independent simulations

properties such as radiosensitivity and host responses such as angiogenesis or immunoediting [14, 21], leads to the establishment of highly patient-specific circumstances, which can greatly affect clinical response. Tumors growing in tissues are faced with harsh biological and chemical conditions as well as physical forces, which all influence the maximum tumor volume that can be achieved in the current condition - the tumor carrying capacity. As a tumor population approaches its carrying capacity, overall proliferation rate saturates dependent on patient specific tumor history. As such, nominal tumor size alone is insufficient to predict growth dynamics.

It is conceivable that different tumor growth rates in vitro and in vivo are not primarily cell intrinsic; rather the impact of the in vivo environment may be the dominant mechanism that modulates cell behavior, which is absent when expanded in optimal in vitro conditions. The key feature of the patient-specific volume-tocarrying capacity ratio and the herein proposed proliferation saturation index, PSI, is a uniform cell growth rate as an intrinsic property that is modulated by host tissue conditions. This leads to different tumor population growth rates before, during and after radiation, including accelerated repopulation during radiotherapy. From two temporally separated radiological scans, routinely taken at diagnosis and treatment simulation, the change in individual in vivo tumor volume can be estimated and compared to the expected in vitro tumor growth, which allows for the estimation of the tumor environmentenforced patient-specific proliferation saturation index, PSI, using the analytic solution to the logistic growth model (Eqn. 1). If diagnostic images are unavailable, two

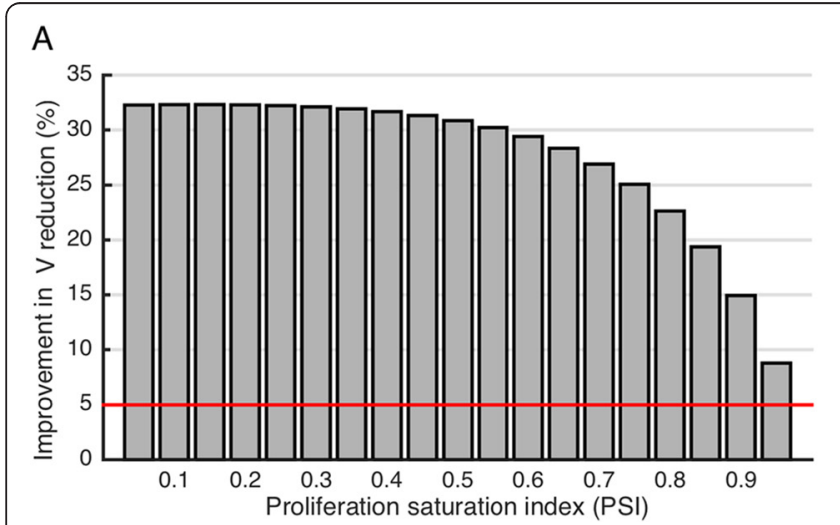

B

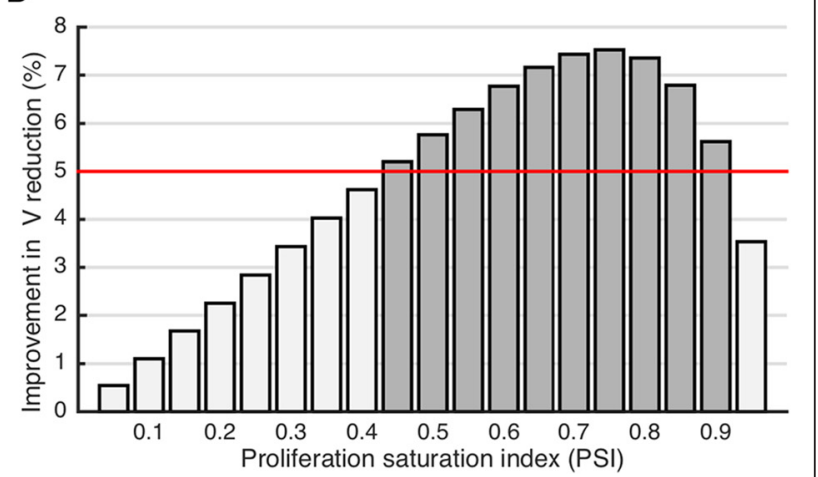

Fig. 4 In silico comparison of altered fractionation regimes using parameters estimated for NSCLC patients. a Model predicted improvement in tumor volume reduction when comparing (1.2 Gy x 58; b.i.d. 9 am and 3 pm; BED=73.8 Gy) RTOG phase III hyperfractionation [36] to the standard of care (2 Gy x 30; q.d. 9 am; BED=66 Gy) as a function of proliferation saturation index (PSI). Red line at 5 \% indicates statistical significance. b Model predicted improved tumor volume reduction when comparing (1.2 Gy $\times 58$; b.i.d. 9 am and 3 pm) hyperfractionation to the daily doses with equal BED (2.21 Gy x 30; q.d. 9 am; BED=73.8 Gy) as a function of proliferation saturation index (PSI) 
subsequent images taken at later time points during therapy may be used to forecast the response to the remainder of the treatment schedule, and to adapt the protocol if necessary.

We introduced a patient-specific tumor carrying capacity in the logistic tumor growth model, and showed that simulations of tumor volume changes during RT using individual pretreatment PSI (equivalent to tumor volumeto-carrying capacity ratio) can reproduce historical radiation response data with high confidence $\left(R^{2}=0.98\right)$. Henceforth, in silico trials may be performed to predict which patients benefit from altered treatment protocols dependent on individual PSI and tumor volumes. The ability to forecast the response of individual tumors to different fractionations may pave the way for clinical trials to recommend either standard of care or alternative radiation fractionation on a per patient basis.

The presented model underestimates radiation-induced cell kill as non-proliferative cells are assumed to be completely radioresistant. Before consequential conclusions on alternative fractionations can be drawn, better approximations of radiation effects on the non-proliferative compartment have to be derived. We have refrained from such considerations in the present study in order to keep the number of unknown parameters small and thus preserve validity of the discussed concept. For simplicity we have limited our analysis to the effects of tumor environment on tumor properties and neglected variability in tumorintrinsic properties. It is conceivable that future studies may integrate patient-specific molecular identifiers of intrinsic radiosensitivity [37], such as RSI [38, 39].

While simulation results using the concept of PSI fit retrospective data with high confidence, additional caution is warranted. The tumor carrying capacity is a dynamic entity that is unlikely to remain constant during the course of radiotherapy. Numerous biological processes such as vascular density, neovascularization, or immune surveillance contribute to variation in the carrying capacity, and the effects of radiation protocols on each of these processes individually and in combination are yet to be fully understood. Whilst high-resolution longitudinal measurements of contributors to carrying capacity, including those presently unidentified, are elusive, frequent tumor volume measurements during fractionated radiotherapy will provide patient-specific data to further fit the tumor growth model. It may also help us develop models that can form the basis for adaptive radiation therapy, which are greatly needed. For example, insights into the evolution of PSI during a course of therapy have the potential to help us understand the predictability of response. With a better understanding of the evolution of such dynamic factors as tumor response and PSI during the initially prescribed radiation protocol, different protocols could be simulated and the treatment protocol dynamically adapted to hopefully provide better outcomes. This will be the subject of future investigations.

\section{Abbreviations}

PSI: Proliferation saturation index; V: Tumor volume; K: Tumor carrying capacity; RT: Radiotherapy; $T_{\text {РОт: }}$ Potential doubling time; $T_{\text {eff: }}$ Effective doubling time.

\section{Competing interests}

The authors declare that they have no competing interests.

\section{Authors' contributions}

SP carried out the mathematical modeling and numerical simulation. JP carried out the mathematical modeling and numerical simulation and helped to draft the manuscript. EM, JC, JFT-R, KL, JKL, RM, LB participated in study design and coordination and helped to draft the manuscript. HE conceived of the study, and participated in its design and coordination and helped to draft the manuscript. All authors read and approved the final manuscript.

\section{Acknowledgements}

This publication is supported by the Personalized Medicine Award 09-33504-14-05 from the DeBartolo Family Personalized Medicine Institute Pilot Research Awards in Personalized Medicine (PRAPM). We thank Rachel Walker for critical reading of the manuscript.

\section{Author details}

${ }^{1}$ Departments of Integrated Mathematical Oncology, H. Lee Moffitt Cancer Center \& Research Institute, 12902 Magnolia Drive, Tampa, FL 33612, USA. ${ }^{2}$ Department of Radiation Oncology, H. Lee Moffitt Cancer Center \& Research Institute, 12902 Magnolia Drive, Tampa, FL 33612, USA. ${ }^{3}$ Department of Cancer Imaging and Metabolism, H. Lee Moffitt Cancer Center \& Research Institute, 12902 Magnolia Drive, Tampa, FL 33612, USA. ${ }^{4}$ Department of Biostatistics and Bioinformatics, H. Lee Moffitt Cancer Center \& Research Institute, 12902 Magnolia Drive, Tampa, FL, USA. ${ }^{5}$ Department of Radiation Oncology, Washington University School of Medicine, St. Louis, MO, USA.

Received: 31 March 2015 Accepted: 16 July 2015

Published online: 31 July 2015

\section{References}

1. Yan D, Lockman D, Brabbins D, Tyburski L, Martinez A. An off-line strategy for constructing a patient-specific planning target volume in adaptive treatment process for prostate cancer. Int J Radiat Oncol Biol Phys. 2000;48:289-302.

2. Woodford C, Yartsev S, Dar AR, Bauman G, Van Dyk J. Adaptive radiotherapy planning on decreasing gross tumor volumes as seen on megavoltage computed tomography images. Int J Radiat Oncol Biol Phys. 2007;69:1316-22.

3. Feng M, Kong F-M, Gross M, Fernando S, Hayman JA, Ten Haken RK. Using fluorodeoxyglucose positron emission tomography to assess tumor volume during radiotherapy for non-small-cell lung cancer and its potential impact on adaptive dose escalation and normal tissue sparing. Int J Radiat Oncol Biol Phys. 2009;73:1228-34.

4. Veiga C, McClelland J, Moinuddin S, Lourenço A, Ricketts K, Annkah J, et al. Toward adaptive radiotherapy for head and neck patients: Feasibility study on using CT-to-CBCT deformable registration for "dose of the day" calculations. Med Phys. 2014;41:031703.

5. Sharma N, Neumann D, Macklis R. The impact of functional imaging on radiation medicine. Radiat Oncol. 2008;3:25.

6. Rockne RC, Trister AD, Jacobs J, Hawkins-Daarud AJ, Neal ML, Hendrickson $K$, et al. A patient-specific computational model of hypoxia-modulated radiation resistance in glioblastoma using 18F-FMISO-PET. J R Soc Interface. 2015;12

7. Bhatnagar $P$, Subesinghe M, Patel C, Prestwich R, Scarsbrook AF. Functional imaging for radiation treatment planning, response assessment, and adaptive therapy in head and neck cancer. Radiographics. 2013:33:1909-29.

8. Clausen MM, Hansen AE, Lundemann M, Hollensen C, Pommer T, Munck Af Rosenschöld P, et al. Dose painting based on tumor uptake of Cu-ATSM and FDG: a comparative study. Radiat Oncol. 2014;9:228. 
9. Ahmed KA, Correa CR, Dilling TJ, Rao NG, Shridhar R, Trotti AM, et al. Altered fractionation schedules in radiation treatment: a review. Semin Oncol. 2014;41:730-50.

10. Prasanna A, Ahmed MM, Mohiuddin M, Coleman CN. Exploiting sensitization windows of opportunity in hyper and hypo-fractionated radiation therapy. J Thorac Dis. 2014;6:287-302.

11. Folkman J, Merler E, Abernathy C, Williams G. Isolation of a tumor fractor responsible for angiogenesis. J Exp Med. 1971; 133:275-288.

12. Kallinowski F, Okunieff P. Blod flow, oxygen and nutrient suppy, and metabolic microenvironment of human tumors: a review. Cancer Res. 1989; 49:6449-6465.

13. Roose T, Netti PA, Munn LL, Boucher Y, Jain RK. Solid stress generated by spheroid growth estimated using a linear poroelasticity model. Microvasc Res. 2003;66:204-12

14. Dunn GP, Bruce AT, Ikeda H, Old LJ, Schreiber RD. Cancer immunoediting: from immunosurveillance to tumor escape. Nat Immunol. 2002;3:991-8.

15. Wilkie KP, Hahnfeldt P. Mathematical models of immune-induced cancer dormancy and the emergence of immune evasion. Interface Focus. 2013:3:20130010-0.

16. Enderling H, Park D, Hlatky LR, Hahnfeldt P. The Importance of Spatial Distribution of Stemness and Proliferation State in Determining Tumor Radioresponse. Math Model Nat Phenom. 2009;4:117-33.

17. Gao X, McDonald JT, Hlatky LR, Enderling H. Acute and fractionated irradiation differentially modulate glioma stem cell division kinetics. Cancer Res. 2013;73:1481-90.

18. Rockne R, Rockhill JK, Mrugala M, Spence AM, Kalet I, Hendrickson K, et al. Predicting the efficacy of radiotherapy in individual glioblastoma patients in vivo: a mathematical modeling approach. Phys Med Biol. 2010;55:3271-85.

19. Folkman J, Hochberg M. Self-regulation of growth in three dimensions. J Exp Med. 1973;138:745-53.

20. Almog N, Henke V, Flores L, Hlatky LR, Kung AL, Wright RD, et al. Prolonged dormancy of human liposarcoma is associated with impaired tumor angiogenesis. FASEB J. 2006;20:947-9.

21. Hahnfeldt P, Panigrahy D, Folkman J, Hlatky LR. Tumor development under angiogenic signaling: a dynamical theory of tumor growth, treatment response, and postvascular dormancy. Cancer Res. 1999;59:4770-5.

22. Freyer JP, Sutherland RM. Regulation of growth saturation and development of necrosis in EMT6/Ro multicellular spheroids by the glucose and oxygen supply. Cancer Res. 1986;46:3504-12.

23. Dale RG, Jones B: Radiobiological Modelling in Radiation Oncology. British Inst of Radiology; 2007.

24. Chougule A, Supe SJ. Linear quadratic model-estimation of alpha/beta ratio for mucosal reaction. Strahlenther Onkol. 1993;169:427-30.

25. Fowler JF. The linear-quadratic formula and progress in fractionated radiotherapy. Br J Radiol. 2014;62:679-94.

26. Yorio JT, Xie Y, Yan J, Gerber DE. Lung cancer diagnostic and treatment intervals in the United States: a health care disparity? J Thorac Oncol. 2009;4:1322-30.

27. Wang P, Feng Y. A mathematical model of tumor volume changes during radiotherapy. Sci World J. 2013;2013:1-5.

28. Park LJ, Park CH, Park C, Lee T. Application of genetic algorithms to parameter estimation of bioprocesses. Med Biol Eng Comput. 1997;35:47-9.

29. Shibamoto Y, Hara M: Radiobiology of normal lung tissue and lung tumours. Med Radiol Radiat Oncol 2005:59-65.

30. Maciejewski B, Withers HR, Taylor JM, Hliniak A. Dose fractionation and regeneration in radiotherapy for cancer of the oral cavity and oropharynx: tumor dose-response and repopulation. Int J Radiat Oncol Biol Phys. 1989;16:831-43.

31. Fowler JF, Chappell R. Non-small cell lung tumors repopulate rapidly during radiation therapy. Int J Radiat Oncol Biol Phys. 2000;46:516-7.

32. Fowler JF. Linear quadratics is alive and well: in regard to Park et al. (Int J Radiat Oncol Biol Phys 2008;70:847-852). Int J Radiat Oncol Biol Phys. 2008;72:957-8

33. Chi A, Wen S, Liao Z, Fowler J, Xu J, Nguyen NP, et al. What would be the most appropriate $\alpha / \beta$ ratio in the setting of stereotactic body radiation therapy for early stage non-small cell lung cancer. Biomed Res Int. 2013;2013:391021.

34. Buch $K$, Peters $T$, Nawroth $T$, Sänger $M$, Schmidberger $H$, Langguth $P$. Determination of cell survival after irradiation via clonogenic assay versus multiple MTT Assay - A comparative study. Radiat Oncol. 2012;7:1.
35. Wee CW, Sung W, Kang H-C, Cho KH, Han TJ, Jeong B-K, et al. Evaluation of variability in target volume delineation for newly diagnosed glioblastoma: a multi-institutional study from the Korean Radiation Oncology Group. Radiat Oncol. 2015;10:137

36. Sause W, Kolesar P, Taylor IV S, Johnson D, Livingston R, Komaki R, et al. Final results of phase III trial in regionally advanced unresectable non-small cell lung cancer: Radiation Therapy Oncology Group, Eastern Cooperative Oncology Group, and Southwest Oncology Group. Chest. 2000;117:358-64.

37. Unger K. Integrative radiation systems biology. Radiat Oncol. 2014;9:21.

38. Eschrich SA, Pramana J, Zhang H, Zhao H, Boulware D, Lee J-H, et al. A gene expression model of intrinsic tumor radiosensitivity: prediction of response and prognosis after chemoradiation. Int J Radiat Oncol Biol Phys. 2009:75:489-96.

39. Eschrich SA, Fulp WJ, Pawitan Y, Foekens JA, Smid M, Martens JWM, et al. Validation of a radiosensitivity molecular signature in breast cancer. Clin Cancer Res. 2012;18:5134-43.

\section{Submit your next manuscript to BioMed Central and take full advantage of:}

- Convenient online submission

- Thorough peer review

- No space constraints or color figure charges

- Immediate publication on acceptance

- Inclusion in PubMed, CAS, Scopus and Google Scholar

- Research which is freely available for redistribution 